\title{
Extracellular Signals That Regulate the Tangential Migration of Olfactory Bulb Neuronal Precursors: Inducers, Inhibitors, and Repellents
}

\author{
Heather A. Mason, ${ }^{1}$ Susumu Ito, ${ }^{2}$ and Gabriel Corfas ${ }^{1}$ \\ ${ }^{1}$ Division of Neuroscience and Department of Neurology, Children's Hospital and Harvard Medical School, Boston \\ Massachusetts 02115, and 2Department of Neurobiology, Harvard Medical School, Boston, Massachusetts 02115
}

Neuronal migration is an essential developmental step in the construction of the vertebrate nervous system, but the extracellular signals involved in initiating and regulating neuronal movement remain unclear. Here we report the identification of a novel astrocyte-derived migration-inducing activity (MIA). Using an in vitro assay, we show that MIA induces the migration of olfactory bulb interneuron precursors, increasing the number of migrating cells and the distance they move. We established quantitative criteria to distinguish between the biological effects of inducers, inhibitors, repellents, and attractants on migrating cells and used them to compare the effects of MIA with those of Slit, a putative repulsive guidance cue. Our analysis demon- strates that, by themselves, MIA induces and Slit inhibits migration from subventricular zone explants. However, when presented together with MIA, Slit acts as a repellent. This study shows that glial cells play a critical role in initiating and modulating the movement of neuronal precursors through the release of a diffusible protein. Moreover, this study provides evidence that the guidance of migrating neuronal precursors is an integrative process, resulting from the cooperation of distinct extracellular factors, and that the function of Slit is context dependent.

Key words: neuronal migration; chain migration; olfactory bulb; subventricular zone; Slit; astrocytes
Neuronal migration, the movement of neuronal precursors from their birthplaces in the proliferative layers to their ultimate locations in the mature brain, is a critical step in the development of the vertebrate nervous system. Neuronal migration is most likely regulated by different types of extracellular signaling molecules that cells encounter as they migrate. Identification of these signals would illuminate the mechanisms involved in the construction of the nervous system and the mechanisms of pathogenesis of brain diseases in which migration has been implicated, such as epilepsy, schizophrenia, and mental retardation (Aicardi, 1994; Raymond et al., 1994; Jones, 1995; Uher and Golden, 2000).

The migration of interneurons from the anterior subventricular zone (SVZ) (Luskin, 1993) to the olfactory bulb provides a useful system to study the cellular and molecular mechanisms that regulate neuronal migration. These neuronal precursors migrate tangentially through a distinct pathway within the SVZ called the rostral migratory stream (RMS), and this migration occurs throughout life (Lois and Alvarez-Buylla, 1994). Migrating cells in the RMS are closely associated with one another, forming elongated cell aggregates resembling chains (Rousselot et al.,

Received Feb. 15, 2001; revised June 21, 2001; accepted July 17, 2001.

This research was supported in part by National Institute of Neurological Disorders and Stroke Grant R01 NS35884 (G.C.), The Klingenstein Foundation (G.C.), The EJLB Foundation (G.C.), the National Alliance for Research on Schizophrenia and Depression (G.C.), and Mental Retardation Research Center/National Institutes of Health Grant P30-HD 18655 (G.C.). We thank Yi Rao for providing the $\mathrm{x}$-Slit expressing cells, Marc Tessier-Lavigne for the Slit and Netrin antibodies, Connie Cepko for providing growth factors, Anthony Frankfurter for the gift of the TuJ1 antibody, Jennifer Marler for her help with the myocultures, and Magdi Sobeih for performing the Western blot analysis. We thank Michael Greenberg, Tom Schwarz, and Zhigang He for their useful comments on this manuscript.

Correspondence should be addressed to Gabriel Corfas, Division of Neuroscience, Children's Hospital, 300 Longwood Avenue, Boston, MA 02115. E-mail: gabriel.corfas@tch.harvard.edu.

Copyright (ㄷ) 2001 Society for Neuroscience $\quad 0270-6474 / 01 / 217654-10 \$ 15.00 / 0$
1995). Three-dimensional reconstruction by serial section electron microscopy and immunocytochemistry revealed that astrocytic processes surround migrating neuroblasts, forming "tubes" around them (Jankovski and Sotelo, 1996; Lois et al., 1996; Peretto et al., 1997). The migrating neuroblasts make contacts with one another, forming specialized zonula adherens-like junctions (Lois et al., 1996). Because these junctions are not observed between neuroblasts and surrounding glia, it has been proposed that neuroblasts migrate along each other rather than on the ensheathing astrocytes (Lois et al., 1996). The role of astrocytes in the migration of these neuronal precursors remains unclear (O’Rourke, 1996).

Several extracellular molecules are involved in neuronal migration in the RMS. Mice lacking the polysialyated form of the neural cell adhesion molecule (PSA-NCAM) have reduced olfactory bulbs, presumably attributable to reduced migration (Tomasiewicz et al., 1993; Chazal et al., 2000), and enzymatic removal of polysialic acid (PSA) from NCAM in SVZ explants results in decreased migration (Ono et al., 1994; Hu et al., 1996). In addition, altering Eph receptor tyrosine kinase signaling appears to disrupt the proliferation and migration of neuroblasts in the SVZ (Conover et al., 2000). Finally, Slit, a secreted protein that interacts with Robo receptors (Brose et al., 1999; Li et al., 1999), has been proposed to act as a repellent for migrating SVZ cells, because cells from SVZ explants migrate away from localized sources of Slit (Hu, 1999; Wu et al., 1999; Chen et al., 2001).

To further characterize the cellular and molecular mechanisms that regulate neuronal chain migration and to determine the role of glia in this process, we studied the migration of olfactory bulb neuronal precursors in vitro. We developed a cell culture paradigm in which neuronal precursors migrate from SVZ explants in chains that are remarkably similar to those observed in vivo. Using this system, we found that astrocytes release a soluble 
protein called MIA, for migration-inducing activity, which induces neuronal migration. Moreover, we found that Slit, a protein reported to repel migrating cells, is a potent inhibitor of migration. Quantitative analyses of the effects of Slit and MIA show that neither of these molecules act primarily as a chemoattractant or chemorepellent, but it is only their combination that leads to repulsion. Thus, the biological context determines how different types of regulatory molecules affect migrating cells. This study demonstrates a previously unknown function for glia in initiating and enhancing the movement of neuronal precursors and suggests that the repulsion of migrating cells within the RMS results from cooperation between distinct extracellular factors.

\section{MATERIALS AND METHODS}

SVZ explant cultures. Olfactory bulbs from postnatal day 3 (P3) to $\mathrm{P} 7$ Long-Evans rats were removed in ice-cold PBS and sectioned coronally $(200 \mu \mathrm{m})$ with a tissue chopper (Stoelting Inc., Kiel, WI). From each section, the SVZ was identified by its translucent appearance, dissected, and cut into several pieces. Explants were subsequently plated on tissue culture dishes coated either with poly-D-lysine (PDL) $(25 \mu \mathrm{g} / \mathrm{ml}$; Collaborative Research, Bedford, MA) or in gels of Matrigel (Becton Dickinson, Cockeysville, MD) diluted 1:2 in DMEM-F12 medium (Life Technologies, Gaithersburg, MD) supplemented with penicillin/streptomycin, and L-glutamine. The medium was supplemented with either $10 \%$ serum (5\% fetal bovine serum and 5\% horse serum) or serum-free additives (N2 or B27; Life Technologies). Care and handling of animals was in accordance with institutional guidelines.

Ultrastructural analysis. Forty-eight hours after plating, cultures were fixed in $2.5 \%$ glutaraldehyde, $1.25 \%$ formaldehyde, and $0.03 \%$ picric acid in $0.1 \mathrm{M}$ cacodylate buffer, $\mathrm{pH}$ 7.2. Specimens were embedded in a mixture of Epon and Araldite, and light gold thin sections were stained with uranyl acetate and lead citrate. Photographs were obtained using a Jeol (Peabody, MA) 1200EX electron microscope.

Immunocytochemistry. SVZ explants and chains were fixed for $10 \mathrm{~min}$ in $4 \%$ paraformaldehyde, rinsed in PBS, blocked, and permeabilized with $3 \%$ BSA plus $0.1 \%$ Triton X-100 in PBS for $1 \mathrm{hr}$ at room temperature and incubated with primary antibodies. Two monoclonal antibodies against neuron-specific class III $\beta$ tubulin [TuJ1 and class III $\beta$ tubulin from Sigma (St. Louis, MO)] were used to identify neuronal precursors $\left(1: 1000\right.$ in $3 \%$ BSA plus $0.1 \%$ Triton X-100 in PBS overnight at $\left.4^{\circ} \mathrm{C}\right)$. Rabbit anti-GFAP antibodies (Dako, High Wycombe, UK) were used to identify astrocytes (1:500 in 3\% BSA plus $0.1 \%$ Triton X-100 in PBS overnight at $4^{\circ} \mathrm{C}$ ). Samples were then washed with PBS and incubated with anti-mouse Cy3 (1:500 dilution) and anti-rabbit IgM-BODIPY (1:250 dilution) secondary antibodies (Molecular Probes, Eugene, OR) for $1 \mathrm{hr}$ at room temperature. Nuclei were stained with Hoescht 33342 (Molecular Probes). Samples were washed in PBS, mounted in 1:1 PBS/glycerol, and photographed on an inverted microscope (Eclipse TE300; Nikon, Tokyo, Japan).

Cell culture. Cerebellar granule neurons and astrocytes were purified from P4-P6 rats as described by Rio et al. (1997). Astrocytes from the SVZ were isolated by removing olfactory bulbs from P3-P6 rats, hemisecting them longitudinally, and dissecting out the SVZ. The tissue was treated with trypsin $(1 \mathrm{mg} / \mathrm{ml}$; Sigma $)$ for $30 \mathrm{~min}$ at $37^{\circ} \mathrm{C}$ and then triturated with fire-polished glass pipettes in HBSS containing $2 \mathrm{mg} / \mathrm{ml}$ BSA, $1 \mathrm{mg} / \mathrm{ml}$ DNase, $30 \mathrm{~mm}$ glucose, and $1 \mathrm{mg} / \mathrm{ml}$ soybean trypsin inhibitor (STI). Dissociated cells were plated on plates coated with PDL $(25 \mu \mathrm{g} / \mathrm{ml})$. Cells were passaged two times to remove any contaminating neurons and identified as glia by GFAP staining. P4 rat myoblasts were purified according to the protocol of Rando and Blau (1994). P4 rat skin fibroblasts were dissociated by treatment with collagenase-dispase at $37^{\circ} \mathrm{C}$ for $30 \mathrm{~min}$, followed by trituration with fire-polished glass pipettes.

Preparation of conditioned media. DMEM-F12 medium was conditioned for $48 \mathrm{hr}$ on $75 \%$ confluent primary cell cultures. The following media were conditioned: medium alone; medium supplemented with 5\% fetal bovine serum and 5\% horse serum; medium supplemented with B-27 (Life Technologies); or medium supplemented with N2 (Life Technologies). Media were then filtered with $0.2 \mu \mathrm{m}$ filters and added to SVZ explant cultures after 2:1 dilution with the appropriate fresh medium. For explants cultured in Matrigel, equal parts of Matrigel, fresh medium, and concentrated conditioned medium (CM) were added. For concentrated conditioned medium 10 and $50 \mathrm{kDa}$ molecular weight cutoff concentrators (Amicon, Beverly, MA) were used.
Trypsinization of astrocyte-conditioned medium (ACM) was achieved by adding $0.5 \mathrm{mg} / \mathrm{ml}$ trypsin (Worthington, Freehold, NJ) to ACM, incubating for $30 \mathrm{~min}$ at $37^{\circ} \mathrm{C}$, and finally adding $1 \mathrm{mg} / \mathrm{ml} \mathrm{STI} \mathrm{(Worth-}$ ington). In control samples, STI was added just before trypsin and incubated as above. Samples were then filtered using $0.2 \mu \mathrm{m}$ filters and added to SVZ explants diluted 2:1 with fresh medium.

SVZ-astrocyte monolayer cocultures. For SVZ explant-astrocyte cocultures on PDL-coated plastic, astrocytes purified from the SVZ or cerebellum were plated as a monolayer at densities of 10,000 or 50,000 cells per well in 24-well tissue culture dishes (Costar, Cambridge, MA). After $24 \mathrm{hr}, \mathrm{SVZ}$ explants were added to the astrocyte cultures, and migration distances were measured after appropriate incubation times.

Analysis of migration on PDL. Migration was quantified by measuring the maximum distance that cells had moved away from the perimeter of each explant at 1 or $2 \mathrm{~d}$ in vitro (DIV), depending on the experiment. At least 20 explants were used for each treatment.

SVZ aggregate cocultures in Matrigel. Aggregates were prepared from cerebellar astrocytes, control human embryonic kidney-293 (HEK), and HEK cells expressing Xenopus-Slit (x-Slit) cells by resuspending $10^{6}$ cells in $180 \mu \mathrm{l}$ of Matrigel (diluted previously 1:2 with fresh medium). Each aggregate was plated (six-well plates; Costar) and incubated overnight. The following day, SVZ explants were dissected and placed in fresh Matrigel surrounding the previously plated aggregates. Migration was analyzed between 8 and $24 \mathrm{hr}$ after the SVZ explants were plated. Because Slit has been reported to be effective at repelling cells only over short distances, explants located $1 \mathrm{~mm}$ or closer to aggregates were analyzed. Explants and the cells migrating from them were visually hemisected into proximal and distal halves relative to their location to aggregates. Using the $20 \times$ objective, cells were counted from the proximal and distal halves of the explants. Astrocytes were maintained in culture for no more than 2 weeks after initial purification because, at later times, their migration-inducing activity was greatly reduced.

\section{RESULTS}

\section{Chain migration from SVZ explants}

When SVZ explants from the olfactory bulbs of early postnatal rats were plated onto PDL-coated tissue culture dishes in serumcontaining medium, migrating cells could be observed exiting most explants within a few hours of plating. Forty-eight hours later, migrating cells were organized in chains up to $400 \mu \mathrm{m}$ long that displayed a remarkably constant width (4-20 cells) (Fig. 1 $A$ ). The chains emanating from the SVZ explants shown in Figure $1 A$ were examined with an electron microscope. As observed in vivo (Doetsch and Alvarez-Buylla, 1996; Lois et al., 1996; GarciaVerdugo et al., 1998), primarily two types of cells, neuroblasts (type A) and glia (type B), were identified in the chains in vitro. Type A, with electron-dense cytoplasmic staining, was the most abundant type (Fig. 1B,C, all unlabeled cells). Type B cells, the glial cells with electron-lucent cytoplasmic staining, were seen in all sections but with lower frequency (Fig. $1 B, C$ ). To confirm the identity of the migrating cells, we stained the chains at different times, from 1 to 4 DIV, with cell-specific antibodies. Antibodies against the neuron-specific class III $\beta$ tubulin isotype stained almost all cells in the chains and in the main bodies of the explants at all time points (Figs. 2, 3). Although EM analysis indicated that glial cells might be in the chains as early as $48 \mathrm{hr}$ after plating, strong GFAP staining was only observed in the tissue after 3 DIV, suggesting that the early glial cells (type B) are astrocyte precursors. The number of GFAP-positive cells was small, but they formed a complex network of processes within the chains (Fig. 2).

\section{Screening known factors for modulation of neuronal migration from SVZ explants}

In an effort to identify signals that may regulate neuronal migration, we screened numerous candidate growth factors and drugs 
$\mathbf{A}$

Figure 1. Neuronal chain migration from SVZ explants. $A$, Explants from the SVZ of the olfactory bulb of $\mathrm{P} 3$ rats were cultured for $2 \mathrm{~d}$ on poly-D-lysine-coated tissue culture dishes in medium containing $10 \%$ serum. Arrowheads point to the chains of migrating cells, and arrows mark cells migrating in "sheets." Scale bar, $300 \mu \mathrm{m}$. The explants were fixed, and the chains were processed for transmission electron microscopy. Longitudinal $(B)$ and cross-sectional $(C)$ views were examined. Two cells types were detected: cell type A (not labeled), the most abundant, with electron-dense nuclei that are presumably neuroblasts; cell type B, with electronlucent nuclei that are presumably glia. Scale bar, $4 \mu \mathrm{m}$.

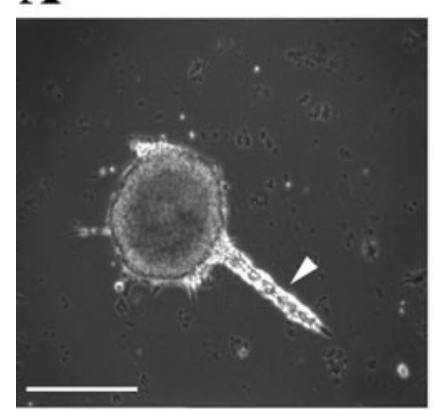

\section{B}

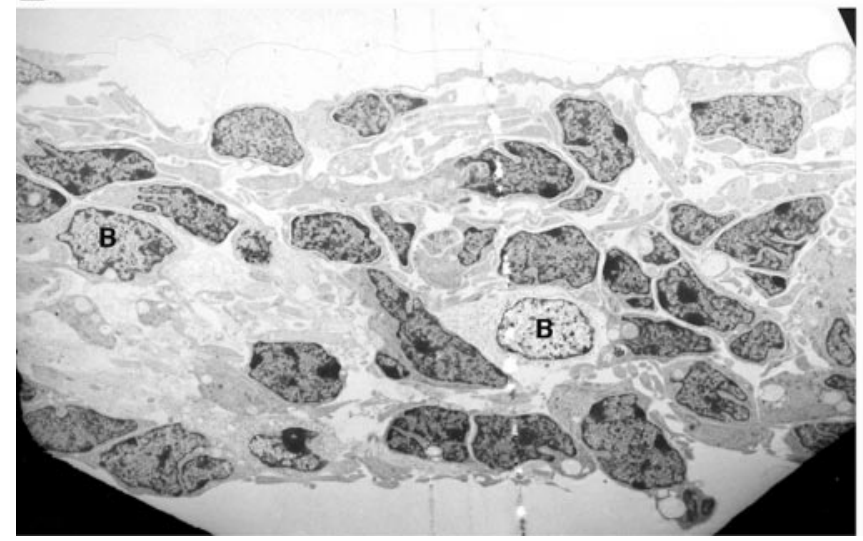

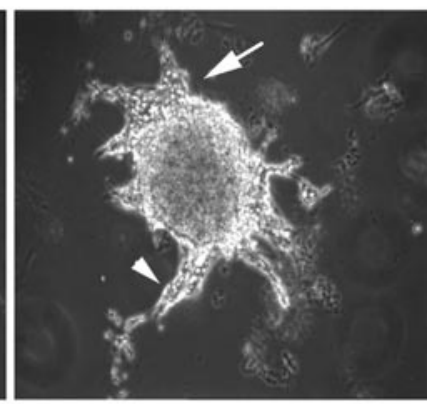

C

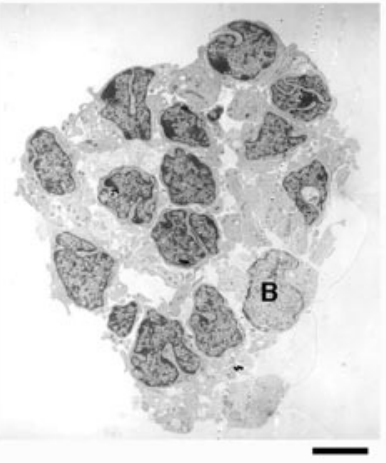

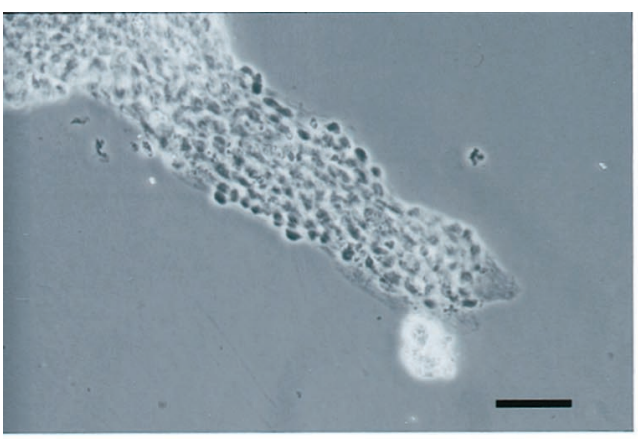
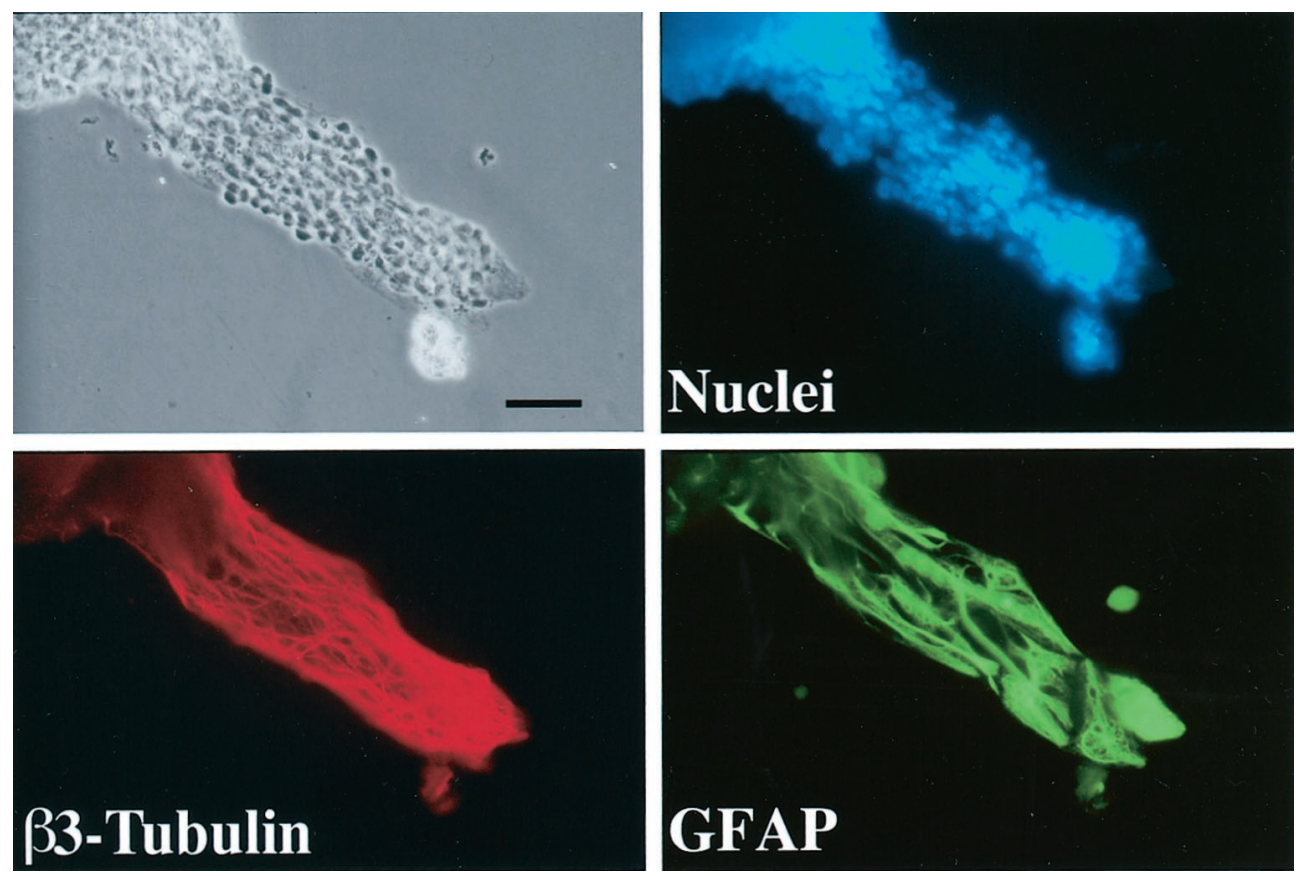

Figure 2. Chains contain neuronal precursors and astrocytes. SVZ explants were cultured for $4 \mathrm{~d}$, fixed, and stained with a nuclear dye (Hoescht 33342) and antibodies against class III $\beta$ tubulin and anti-GFAP antibodies. Most of the cells in the chains are neuronal precursors (class III $\beta$ tubulin-positive), but networks of GFAP-positive fibers are also present in the chains. Scale bar, $60 \mu \mathrm{m}$.

for their ability to enhance or inhibit neuronal migration (Table 1). We tested the involvement of several molecules that have been implicated in neuronal migration, including neuregulin (Anton et al., 1997; Rio et al., 1997), neurotrophins (Brunstrom et al., 1997), PDGF (Forsberg-Nilsson et al., 998), and netrin (Bloch-Gallego et al., 1999; Yee et al., 1999; Alcantara et al., 2000). We also considered as candidates factors that, together with their receptors, are expressed in the RMS, e.g., hepatocyte growth factor/ scatter factor (HGF/SF) (Thewke and Seeds, 1996). Some of these factors had effects on the SVZ explants similar to those reported for other cell types. For example, HGF/SF induced neurite outgrowth from cells in the SVZ explants (data not shown), which has also been observed in spinal cord and neocortical explants (Ebens et al., 1996; Hamanoue et al., 1996). None of the factors tested possessed migration-inducing activity or blocked migration. Although our screen was not exhaustive, several candidate molecules can be excluded as inducers of neuronal migration in this assay. 
$\mathbf{A}$

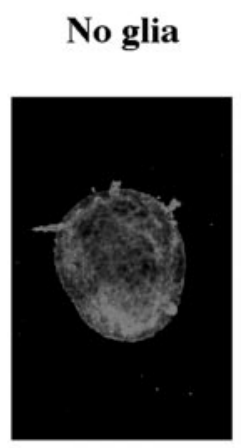
10,000 glia/well

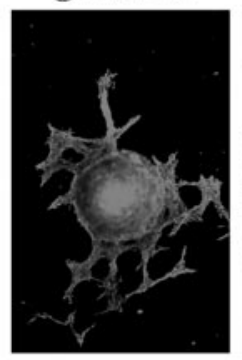

B

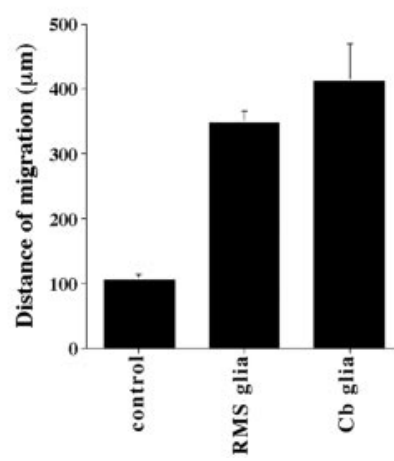

Figure 3. Astrocytes promote neuronal migration from SVZ explants. $A$, SVZ explants were plated on tissue culture dishes alone or in the presence of glia $(10,000$ or 50,000 astrocytes per well). After 1 DIV, explants were fixed and labeled with antibodies against class III $\beta$ tubulin. Scale bar, $100 \mu \mathrm{m}$. $B$, The mean maximum distance of migration was calculated for SVZ explants in the absence or presence of astrocytes purified from either the RMS or cerebellum. In the presence of 50,000 astrocytes per well, cells from SVZ explants moved more than three times as far as cells in the absence of additional glia $(p<0.0001)$. Glia purified from either the RMS or cerebellum $(C b)$ are equally capable of promoting migration from SVZ explants. The error bars represent SEM.
Table 1. Factors and drugs tested for their ability to alter migration from SVZ explants on PDL-plated dishes

Factors tested in migration on PDL Concentration

Bone morphogenic protein-7

Ciliary neurotrophic factor

Brain-derived neurotrophic factor

Neurotrophin-4

Epidermal growth factor

Endothelial cell growth factor

Keratinocyte growth factor

Glial-derived neurotrophic factor

Granulocyte colony stimulating factor

Granulocyte-macrophage colony

stimulating factor

Hepatocyte growth factor/scatter factor

Leukocyte inhibitory factor

Insulin-like growth factor-1

Platelet-derived growth factor B/B

Retinoic acid

Stem cell factor

Transforming growth factor- $\alpha$

Transforming growth factor- $\beta 1$

Tumor necrosis factor- $\alpha$

Leupeptin

High potassium $(\mathrm{KCl})$

Tetraethylammonium

2-Amino-5-phosphono-valerate

Tetrodotoxin

$\omega$-Conotoxin

Forskolin

Pertussis toxin

All treatments were tested for changes in migration in serum-containing and serumfree medium (1 and 2 DIV). None of the treatments led to significant changes in migration.

\section{Astrocytes enhance the migration of neuronal precursors from SVZ explants}

Because none of the molecules tested appeared to have the ability to influence chain migration in vitro, we searched for a physiologically relevant source of such an activity. Most neuronal migrations occur in close association with glial cells, and we showed previously that radial glia play an active role in facilitating the movement of neurons along their surface (Rio et al., 1997). Therefore, we tested whether purified astrocytes affected neuronal migration from SVZ explants. Cocultures of SVZ explants with astrocytes purified from either the RMS or the cerebellum showed that astrocytes dramatically affect migration (Fig. 3). In a dose-dependent manner, astrocytes from both the RMS $(n=54$ explants) and the cerebellum ( $n=21$ explants) lead to an increased number of migrating SVZ cells and greatly enhanced the distance these cells migrated compared with SVZ explants cultured alone ( $n=41$ explants). The effect of the astrocytes was fast, leading to the migration of large numbers of neurons in $<24$ $\mathrm{hr}$, a time when little migration was seen under normal conditions (Fig. 3A).

\section{Astrocyte-conditioned medium promotes the migration of SVZ neuronal precursors}

It was apparent that direct contact between SVZ explants and astrocytes was not required for increased neuronal migration because the increase occurred even when astrocytes were placed at a distance from the explants. This suggested that astrocytes might enhance migration through the release of a soluble factor. Therefore, we tested the ability of ACM to influence neuronal migration under different culture conditions. First, SVZ explants were cultured in serum-free medium, conditions that do not support migration, and were treated with serum-free ACM. Whereas very few explants in defined medium showed neuronal migration, addition of ACM resulted in a 10-fold increase in the probability of migration, with most explants displaying robust migration (Fig. 4A). Several lines of evidence suggest that the lack of migration in serum-free medium was not the result of reduced cell viability. First, when explants were maintained in serum-free medium for 1 week, cells within the explants continued to proliferate (data not shown). Second, explants displayed extensive neurite outgrowth after several days in culture (data not shown). Third, cells from explants plated directly in serum-free medium could be induced to migrate by adding serum $2 \mathrm{~d}$ after plating. In this case, the percentage of explants with migrating cells $48 \mathrm{hr}$ after the addition of serum was similar to that observed from explants plated directly in serum and scored after 2 DIV $(75 \% ; n=64)$.

We then tested the effects of ACM on explants cultured in medium containing $10 \%$ serum to determine whether the activity in ACM regulated other aspects of migration besides initiation. Under these conditions, almost all explants displayed migration in both the absence or presence of ACM, but treatment with ACM led to a dramatic increase in the distance cells migrated (Fig. $4 B$ ) and in the number of migrating cells. The effects of ACM on migration were dose dependent, and maximal activity resulted in a twofold increase in the distance cells migrated away from the explants. 
Figure 4. Astrocytes release a trypsinsensitive factor that induces and increases neuronal migration. $A$, ACM increases the percentage of SVZ explants with migrating cells in serum-free conditions after 2 DIV $(p<0.0001 ; 11$ independent experiments). $B$, Medium conditioned by astrocytes $(A C M)$ but not by cerebellar granule cells $(G C M)$, fibroblasts $(F C M)$, or myotubes (MCM) increases the distance of migration from SVZ explants after 1 DIV $(p<0.012$ for comparison of ACM with all other treatments; averages from 3 independent experiments). $C$, The migration-inducing activity present in ACM is abolished by trypsinization $(p<$ $0.004)$. When STI was added before trypsin, the increase in neuronal migration from SVZ explants was equal to that in untreated ACM ( $p=0.55$; averages from 3 independent experiments). The error bars represent SEM.

\section{A}

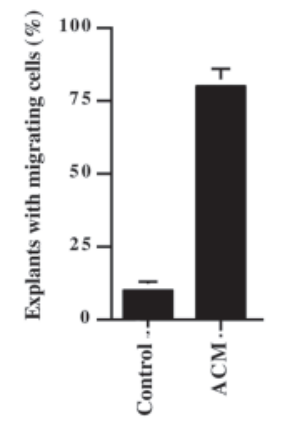

$\overline{\text { Serum-free }}$ medium
B

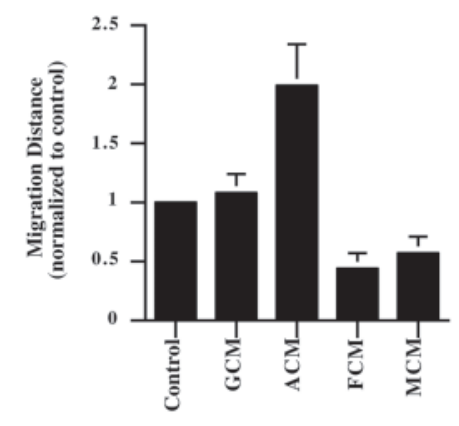

C

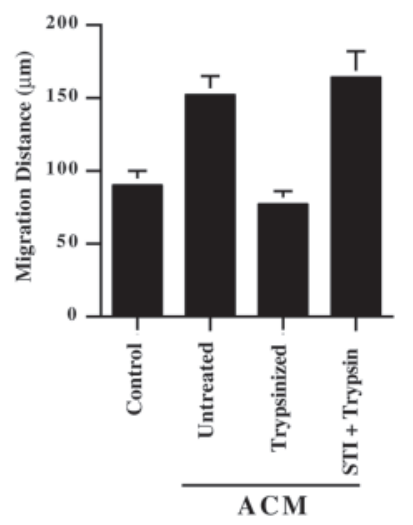

Because serum alone was sufficient to support migration, it was important to determine whether ACM contained the same activity as serum. To test this possibility, we measured the distance of migration in medium containing different serum concentrations $(5,10$, and $20 \%)$ and found the same level of migration under all conditions. Addition of ACM enhanced migration independent of the serum concentration (data not shown). We therefore concluded that the activity in ACM is distinct from factors present in serum. Moreover, only ACM $(n=125)$, not medium conditioned by rat cerebellar granule neurons ( $n=61$ explants), fibroblasts ( $n=70$ explants), myotubes $(n=78)$, or HEK-293 cells (see below), resulted in increased migration compared with untreated control explants $(n=101)$ (Fig. $4 B)$.

To characterize the nature of the astrocyte-derived molecule(s) that modulates neuronal migration, we tested whether the activity in ACM can be destroyed by protease treatment. Serumfree ACM was treated with trypsin, and after addition of STI, this medium was combined with fresh serum-containing medium and added to explants. As a control, the STI was added to the CM before the trypsin. Trypsinization completely abolished the migration-inducing activity in ACM (total number of explants: control, 71; untreated ACM, 91; trypsinized ACM, 89; STI plus trypsin plus ACM, 91) (Fig. 4C), indicating that the activity reflects the function of a protein. Furthermore, by ultrafiltration we found that the molecule responsible for the activity appears to be larger than $50 \mathrm{kDa}$. Together, these results suggest that astrocytes release a soluble protein, MIA, of molecular weight larger than $50 \mathrm{kDa}$ that induces and enhances neuronal migration from SVZ explants. Moreover, this activity appears to be specific to astrocytes because it is not found in medium conditioned by other cell types.

\section{The role of MIA in guiding migrating neurons}

The experiments described above suggest that MIA induces neuronal migration and may contribute to the initiation and maintenance of neuronal movement. The pathway olfactoryinterneuron precursors must travel in vivo is long, and it has been proposed that repulsive and attractive signals contribute to their navigation. Therefore, it was important to determine whether MIA also acts as an attractant, regulating the direction of migration. This could be tested by assessing the effects of a point source of MIA on migration from SVZ explants within a threedimensional gel matrix, as has been done for molecules that direct axonal pathfinding (Tessier-Lavigne et al., 1988).

Measurement of the number and distribution of migrating cells

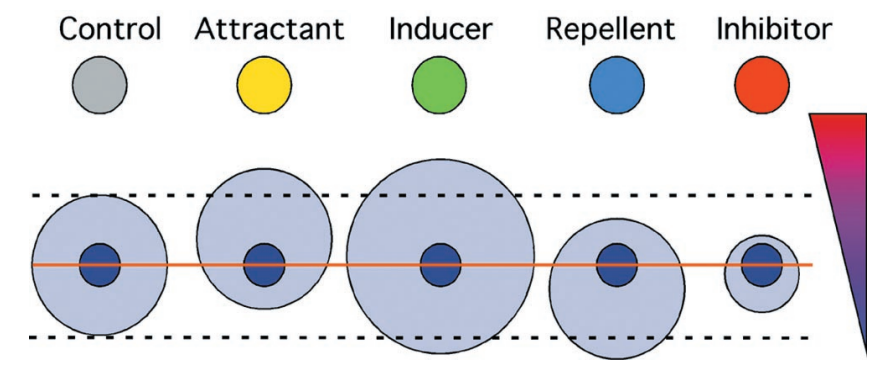

Figure 5. Model of the quantitative and qualitative differences in the effects of attractants, repellents, inhibitors, and inducers on migration from SVZ explants. The top colored circles represent aggregates of cells secreting putative regulators of migration. The small blue circles represent SVZ explants, and the larger light-blue circles represent the migrating cells. The size of the circle signifies the number of cells; their location relative to the inner circle shows the preferred direction of migration. The orange line shows the separation between the distal and proximal hemispheres; the dashed lines facilitate the comparison between control and experimental points. The triangle on the right depicts an expected concentration gradient of the molecules.

should distinguish between four biological effects on migration: induction, inhibition, attraction, and repulsion (Fig. 5). Induction and inhibition should result primarily in a change in the number of migrating cells; attraction and repulsion should lead to a change in their distribution. The effects of an inhibitor and a repellent (or an attractant and an inducer) may be similar in some regards. For example, an inducer and an attractant may lead to more migration from the proximal side. Nevertheless, these activities should be clearly distinguishable by the following criteria. A repulsive signal should decrease migration only toward its source, whereas an inhibitor should decrease migration in all directions; an attractant should increase migration only toward its source, whereas an inducer should increase migration in all directions. Furthermore, the number of cells moving away from a repellent could remain constant or increase, but not decrease. Using these quantitative criteria, we set out to further characterize the effects of MIA and to compare its effects with those of Slit, a putative repulsive guidance cue (Hu, 1999; Wu et al., 1999).

SVZ explants were plated within Matrigel gels in the proximity of aggregates of cerebellar astrocytes, HEK cells, or x-Slit-HEK cells, and the number of migrating cells in the proximal and distal hemispheres were quantified. As reported previously, Slit leads to asymmetric migration, resulting in more cells migrating from the distal side of the explant (Fig. $6 A$ ). In contrast, migration from 
A

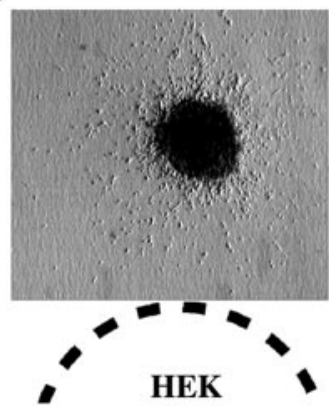

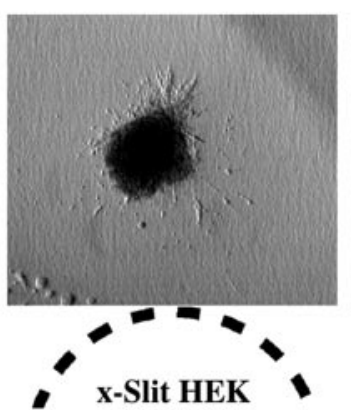

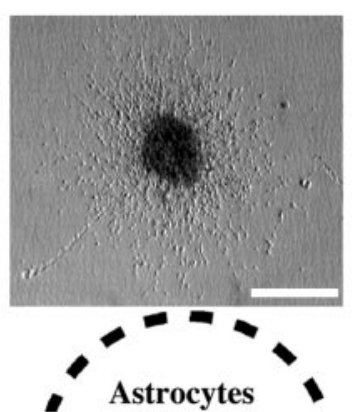

B

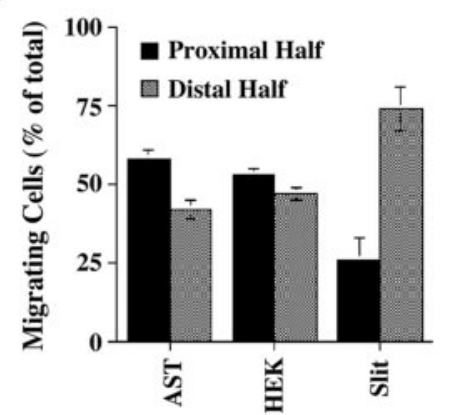

Figure 6. Slit, but not astrocytes, leads to asymmetric migration from SVZ explants. A, SVZ explants were cultured in the proximity of aggregates of HEK cells (right), $\mathrm{x}$-Slit-expressing HEK cells (middle), or astrocytes (left) in Matrigel. The dashed lines indicate the location of the aggregates relative to the explants. Migration appeared asymmetric only in the presence of x-Slit-expressing cells. Scale bar, $300 \mu \mathrm{m}$. B, Quantification of migration from SVZ explants cocultured in the vicinity of aggregates for $11 \mathrm{hr}$ in Matrigel. Although approximately equal numbers of cells migrated from the proximal and distal sides of SVZ explants cultured with HEK and astrocyte aggregates, most of the cells were found migrating from the distal halves of explants cultured with $\mathrm{x}$-Slit-expressing cells.

TOTAL

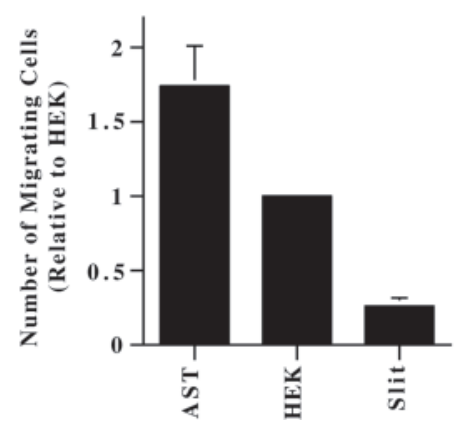

PROXIMAL

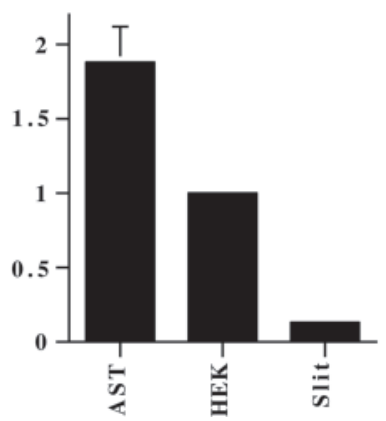

explants in proximity to HEK cells or cerebellar astrocytes appeared symmetric (Fig. 6A). To our surprise, measurement of the number of migrating cells showed that proximity to $\mathrm{x}$-Slitexpressing cells resulted in a reduced number of total migrating neurons $(p \leq 0.02)$ (Fig. 7 , left $)$. The Slit-induced reduction in migration occurred on both sides of the explants $(p<0.04)$, but the decrease was larger in the proximal quadrant (threefold more cells in the distal side; $p=0.0001$ ) (Fig. 7, middle and right). In contrast, proximity to astrocytes resulted in an increase in the total number of migrating cells $(p=0.01)$ (Fig. 7, left), with no significant differences between the proximal and distal sides. (Fig. 7, middle and right). These results are consistent with MIA acting as an inducer and Slit as an inhibitor of migration.

\section{Slit inhibits migration independent of a concentration gradient}

It has been proposed that repulsive guidance cues require a concentration gradient to function. In contrast, an inhibitor should act even when presented in a uniform concentration. To distinguish to what extent Slit functions as an inhibitor of migration rather than as a repellent, we measured the effects of a uniform concentration of Slit protein on the migration from SVZ explants plated in Matrigel gels. Explants were plated in Matrigel gels containing medium conditioned by x-Slit-expressing cells $(n=31)$, and migration was compared with those exposed to HEK-CM $(n=29)$ or ACM $(n=31)$. As expected, migration from the explants was symmetric under all conditions (data not shown). However, the effects of the conditioned media were
DISTAL

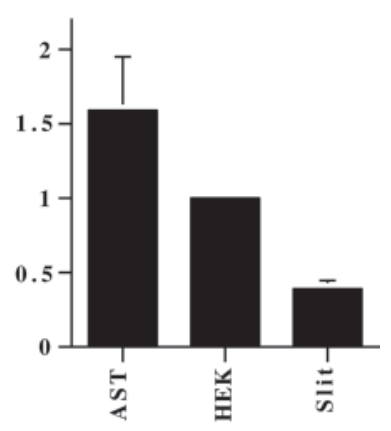

Figure 7. Quantitative analysis of the effects of astrocytes and Slit-expressing cells on neuronal migration from SVZ explants. SVZ explants were cultured in the proximity of aggregates $\left(1 \times 10^{6}\right.$ cells $)$ of HEK cells, x-Slit-expressing HEK cells, or astrocytes for $11 \mathrm{hr}$ in Matrigel. The samples were then fixed, and the number of migrating cells was counted. Coculturing SVZ explants with aggregates of astrocytes increased the total number of migrating SVZ cells, with similar increases from the distal and proximal sides. Conversely, dramatically fewer cells migrated from SVZ explants cocultured with aggregates of $x$-Slit-expressing cells compared with control HEK cells. The effects of Slit on the proximal side were three times larger than in the distal part $(p=0.0001)$.

remarkably similar to the effects observed in the cocultures. Whereas addition of ACM induced an increase in the number of migrating cells, Slit-CM led to a reduction in migration (Fig. 8A). In addition, increasing amounts of Slit-CM resulted in increasingly fewer migrating cells (Fig. $8 B$ ), indicating that the concentration amount of Slit protein determines the level of inhibition. Thus, Slit appears to act as an inhibitor of migration of SVZ cells, and this inhibition occurs independent of a gradient in the concentration of Slit protein.

\section{Slit-induced asymmetry correlates with Slit-induced inhibition of migration}

The experiments described above showed that Slit can lead to inhibition of migration and to repulsion. To determine whether these effects are linked to one another or whether each one is a distinct biological effect of Slit that occurs at different concentrations, we measured the effects of Slit on explants cultured near cell aggregates containing different numbers of Slit-producing cells. Similar to the dose-dependent inhibition observed with Slit-CM, we found that increasing the number of Slit cells in the aggregates (from $5 \times 10^{4}$ to $10^{6}$ cells per aggregate) led to increasingly greater inhibition (Fig. 9A). Even with few Slitexpressing cells in the aggregates $(50,000)$, the total number of cells migrating from SVZ explants was reduced by $>60 \%$. The effects of the aggregates on the direction of migration were also dose dependent, with migration becoming increasingly polarized when more Slit-producing cells were included in the aggregates (Fig. 9B). However, in contrast to the inhibition, the effects of 
Figure 8. Astrocyte-conditioned medium and Slit-conditioned medium exert oppoplants in Matrigel. A, Explants were cultured in Matrigel containing Slit-CM, HEK-CM, or ACM. Migration from SVZ explants was symmetric under all conditions. However, ACM increased and Slit-CM decreased the number of migrating SVZ cells compared with HEK-CM ( $p=0.007$ and $p=0.001$, respectively). $B$, Slit decreases migration from SVZ explants in a dose-dependent manner. SVZ explants were cultured in Matrigel containing different amounts of Slit-CM for 11 hr. The number of cells migrating from SVZ explants was correspondingly reduced with increasing amounts of Slit-CM Maximal levels of Slit resulted in a $>20$ fold decrease in the number of migrating cells compared with control explants. The error bars represent SEM. site effects on migration from SVZ ex-

A

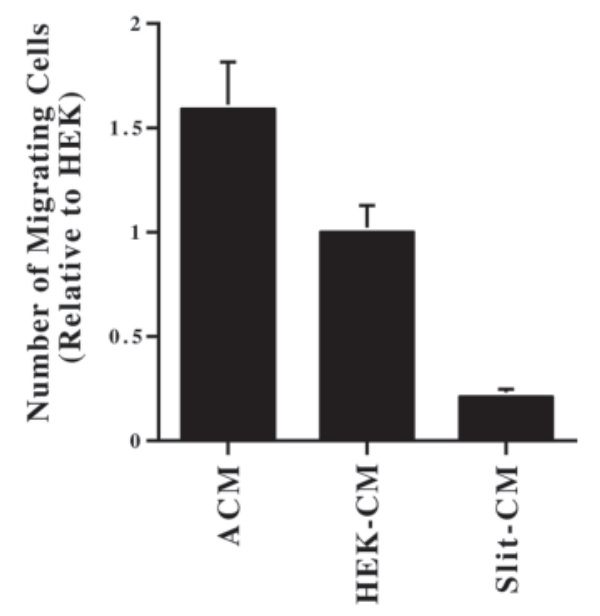

Figure 9. Slit-induced asymmetry corresponds with Slit-induced inhibition of migration. SVZ explants were cultured in Matrigel in the proximity of aggregates that contained the same total number of cells $\left(1 \times 10^{6}\right)$ but a varying proportion of Slit-expressing cells, from $5 \times 10^{4}$ to $1 \times 10^{6}$ cells. Migration from SVZ explants decreases $(A)$ and becomes increasingly asymmetric $(B)$ with the increasing number of Slit cells in the aggregates. The graph depicts data from a representative experiment that was replicated three times. At least 24 explants were used for each treatment. The error bars represent SEM.
A

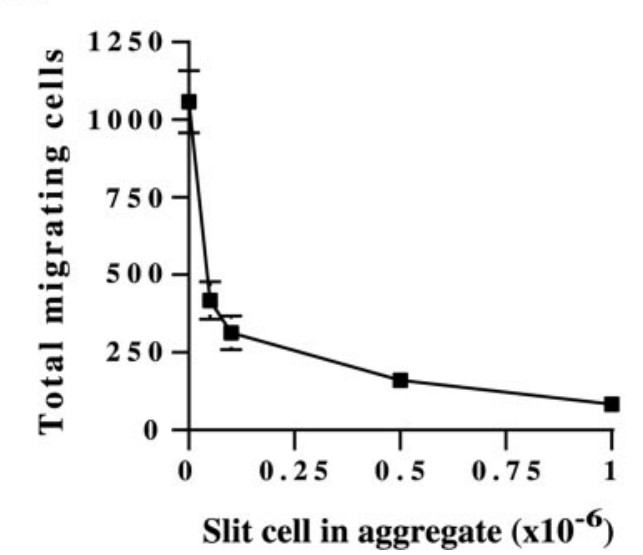

B

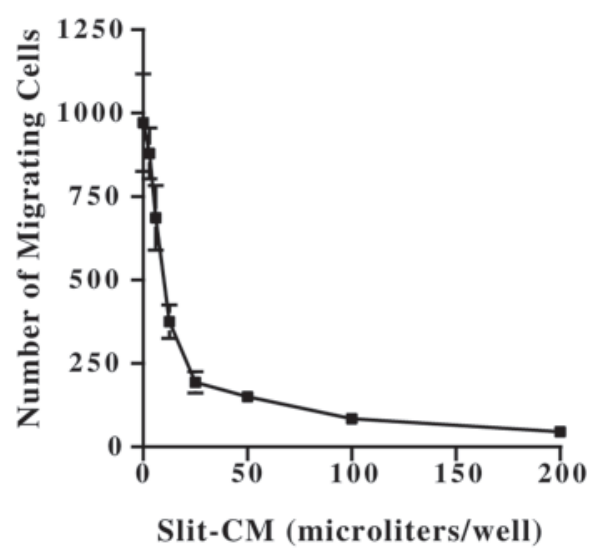

B

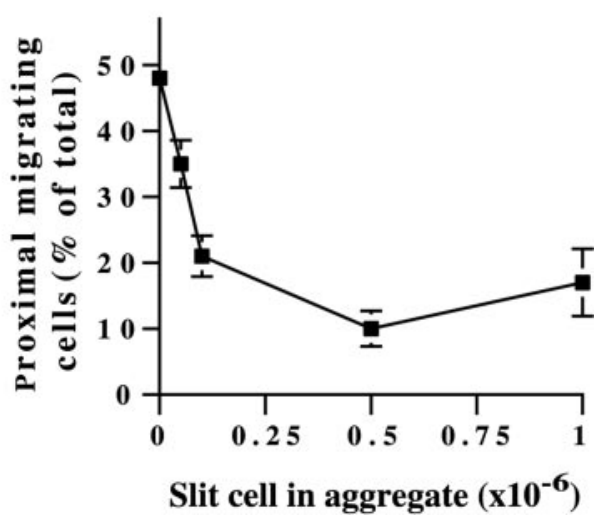

aggregates containing 50,000 Slit-producing cells on the direction of migration were much smaller $(<30 \%)$. Thus, Slit-induced inhibition is more pronounced at lower concentrations than Slitinduced asymmetric migration. These results suggest that Slit acts primarily as an inhibitor of SVZ neuroblast migration and that Slit-mediated asymmetric migration is caused by the different concentrations of Slit on the proximal and distal sides of explants that differentially inhibit cell movement. These findings suggest that directional movement can be generated in response to a localized source of an inhibitor rather than strictly resulting from the action of repellent.

\section{Combination of MIA and Slit in the appropriate spatial configuration leads to directed neuronal migration}

Slit has been reported to be expressed by cells in the septum (Wu et al., 1999), a region that is caudal to the RMS, whereas MIA is secreted by glial cells that are present throughout the migratory pathway. Thus, cells migrating within the RMS would be simultaneously exposed to a uniform concentration of MIA and a posteroanterior decreasing concentration gradient of Slit. We hypothesized that together these molecules might direct neuronal migration from the SVZ to the olfactory bulb in vivo and that the spatial distribution of these signals may be critical for their function.

To test this hypothesis, we compared the behavior of migrating neurons in vitro when presented with a point source of Slit, a uniform concentration of MIA, or simultaneously with a uniform concentration of MIA and a point source of Slit. As in previous experiments, SVZ explants cultured in the presence of Slit aggregates alone displayed reduced migration that was biased away from the source of Slit, whereas explants bathed in ACM alone displayed increased but symmetric migration. Surprisingly, when both MIA and Slit were present, the number of cells migrating from explants was similar to that observed in ACM alone, but most cells migrated away from the Slit source (Fig. 10A). The pattern of migration observed in the combinatory treatment was not attributable to the loss of one of the activities. The degree of asymmetry was similar in explants cultured with Slit aggregates or with Slit aggregates plus ACM (67.3 and 66.1\% migrate from the distal sides, respectively), showing that Slit was active. In addition, the total number of migrating cells was similar to that observed in cultures with ACM alone, showing that MIA was also active (Fig. 10B). Thus, it appears that, in the presence of MIA, Slit acts to redistribute the mass of migrating cells away from the source of Slit without altering the total amount of migration. Moreover, the number of cells migrating from the distal side of explants in the Slit plus ACM conditions was greater than the average number of cells migrating in one hemisphere of explants treated with ACM alone $(p=0.035)$. This suggests that Slit, in the presence of ACM, induces cells that would normally be present on one side of an explant to move to the opposite side. 
A

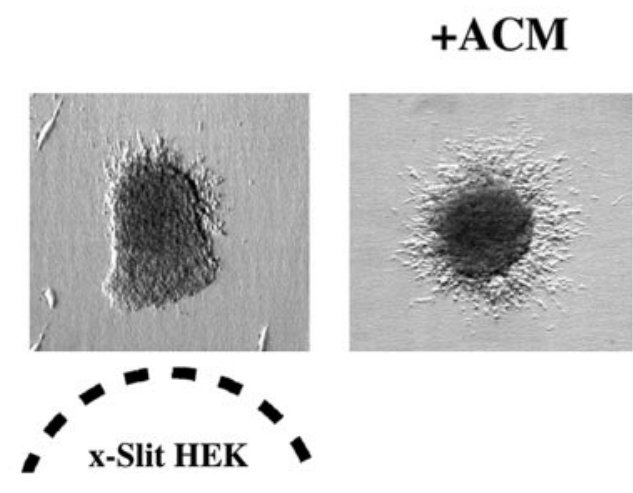

$+\mathrm{ACM}$

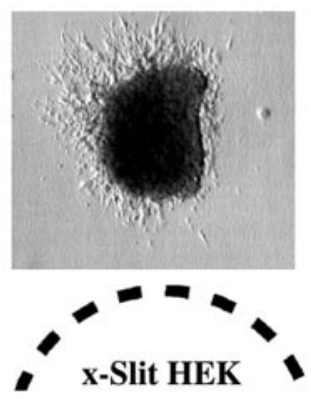

B

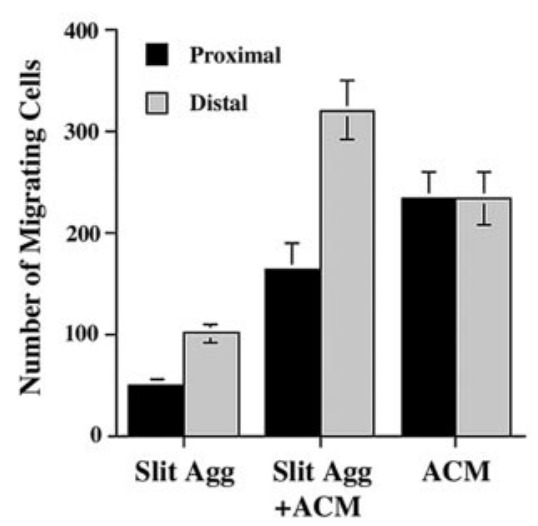

Figure 10. In the presence of ACM, Slit repels migrating neurons from SVZ explants without reducing cell number. $A$, Explants were cultured in Matrigel in the proximity of aggregates of x-Slit-expressing cells (left), Matrigel with ACM (middle), or both (right). The dashed lines indicate the location of the aggregates relative to the explants. Migration was asymmetric in the presence of $x$-Slit-expressing cells. When Slit was presented alone, very few cell migrated, but robust migration away from the Slit source was observed when ACM was also present. $B$, Quantification of migration from explants cocultured near x-Slit-expressing aggregates (Slit Agg), with ACM, or both (Slit Agg $+A C M$ ). The number of cells migrating from the proximal and distal sides of explants plated with Slit aggregates alone were significantly less than those from explants plated with Slit plus ACM. Moreover, the total number of cells migrating in ACM alone or Slit plus ACM was the same, but their distribution was different. Thus, in the presence of ACM, Slit changes the direction of migration without affecting the number of migrating cells.

Thus, the combination of MIA and Slit exert a bona fide chemorepulsive action on SVZ neurons in which the overall amount of migration is constant as well as directed.

\section{DISCUSSION}

Our results show that the migration of RMS neuronal precursors can be regulated in at least three ways, by induction, inhibition, and repulsion, demonstrating that these are distinct biological processes. Furthermore, we identified some signals involved in these processes. Astrocytes stimulate the migration of SVZ cells through the release of a protein-activity (MIA) with an apparent molecular weight $>50 \mathrm{kDa}$, whereas Slit exerts the opposite effect and inhibits their movement. Moreover, these two molecules together, when presented in the appropriate spatial distribution, lead to repulsion. Although it has been suggested that attraction by the olfactory bulb is not involved in the rostral movement of neurons to the bulb (Hu and Rutishauser, 1996; Jankovski et al., 1998; Kirschenbaum et al., 1999), this possibility should be explored further. In vivo, these molecules may regulate neuronal migration in several ways. MIA may help to initiate the movement of precursors away from the germinal layer and to maintain their movement within the RMS. Slit, expressed outside the RMS, may inhibit neuronal movement and thus prevent the migrating cells from entering inappropriate brain regions. Finally, the combined action of both signals within the RMS may bring about proper neuronal migration, by leading to repulsion away from the septum, the source of Slit (Wu et al., 1999).

Our SVZ explants differ from those used by others in that we dissected the tissue from the most anterior part of the RMS in which it enters the olfactory bulb $\left(\mathrm{SVZ}_{\mathrm{ob}}\right)$, whereas other studies obtained the explants from the SVZ bordering the lateral ventricle and septum (SVZ $)$ (Hu and Rutishauser, 1996; Wichterle et al., 1997; Wu et al., 1999). To ensure that our new findings on the effects of Slit on migration are not attributable to differences in the cellular composition of the explants and whether MIA stimulates migration along the entire migratory pathway, we repeated our experiments with $\mathrm{SVZ}_{\mathrm{a}}$ explants. We found that MIA and Slit have the same effects on $\mathrm{SVZ}_{\mathrm{a}}$ and $\mathrm{SVZ}_{\mathrm{ob}}$ explants, indicating that MIA and Slit have the same functions all along this migratory pathway.

Although the identity of MIA remains unknown, our screen has excluded some important candidates. PDGF, which has been shown to induce the movement of cortical neuronal precursors (Forsberg-Nilsson et al., 1998), did not affect migration in our assay. Western blotting showed that the ACM does not contain Netrin-1, a chemoattractant that has been implicated in some forms of nonradial migration (Bloch-Gallego et al., 1999; Yee et al., 1999, Alcantara et al., 2000), and Netrin-1 did not affect migration from explants cultured on PDL. HGF/SF, which has been shown recently to induce the migration of interneurons (Powell et al., 2001), also failed to modulate the migration from SVZ explants on PDL. The data presented above do not rule out the possibility that the activity is attributable to more than one protein, as in the case of the MAX factor, which is the combination of BDNF and neurotrophin-3 (O'Connor and TessierLavigne, 1999). However, preliminary experiments using chromatography suggest that MIA is the result of a single protein.

MIA is produced by astroglia purified from the RMS and the cerebellum, and preliminary experiments show that cortical astroglia also produce it. Moreover, ACM induces the migration of cortical neuronal progenitors in Boyden chambers (M. Sobeih and G. Corfas, unpublished observations) and increases the migration of cerebellar granule neurons on radial glial fibers in vitro (J. M. Peyrin and G. Corfas, unpublished observations). These observations suggest that glial cells, through the release of MIA or similar proteins, directly regulate the migration of all neuronal precursors, including those that move along radial glia.

Our results show that a point source of Slit has two distinct effects on migrating SVZ neuroblasts: (1) the number of migrating cells decreases, and (2) migration becomes asymmetric and biased away from the source of Slit. One explanation for these findings is that Slit is a dual modulator of neuronal movement, capable of both inhibiting and repelling migrating SVZ neuroblasts. An alternative explanation is that Slit functions primarily as an inhibitor of neuronal migration and that asymmetries in cell 
distribution follow as a consequence of the localized differences in its concentration. Several lines of evidence support the latter. First, although many guidance molecules require a concentration gradient to produce an effect, Slit-mediated inhibition occurs even at uniform concentrations in our assay. Second, Slit inhibits migration from SVZ cells in a dose-dependent manner, with increasing amounts of Slit leading to increasingly greater inhibition. Third, Slit-mediated asymmetries in migration are always observed in conjunction with Slit-induced inhibition, whereas inhibition can be seen independently of asymmetry. These data are consistent with a model in which directionality is determined by a gradient in the concentration of a dose-dependent inhibitor.

Additionally, our results show that the effects of Slit on migration depend on the biological context in which it is presented. When presented alone, either in a uniform distribution or in a concentration gradient, Slit inhibits neuronal movement. However, when Slit is presented in conjunction with MIA, repulsion of migrating SVZ neuroblasts is observed. Similarly, several recent studies in axon guidance have highlighted the importance of the interplay between signaling pathways in determining specific cellular responses to guidance cues. For example, the activation of the Slit receptor Robo1 acts to silence the responsiveness of a cell to netrin through the binding of the cytoplasmic domain of Robo1 to the cytoplasmic domain of the netrin receptor DCC (deleted in colorectal cancer) (Stein and Tessier-Lavigne, 2001). In addition, recent studies have demonstrated that the effects of guidance cues can be converted from attractive to repulsive or vice versa by changes in the intracellular levels of cGMP and cAMP (Song et al., 1998). Thus, the actions of extracellular guidance cues are not defined by an inherent activity but by interactions with other extracellular and intracellular signals. Once MIA and its receptor are identified, it will be interesting to determine the interactions between Slit and MIA signaling pathways.

Our in vitro system of explants plated on PDL recapitulates essential features of neuronal chain migration in adult tissues, with neurons migrating in chains containing astrocytes that generate complex networks of processes within the chains. In contrast, when plated in Matrigel, most chains have a one to two cell diameter (what we call "single-file" migration), many neurons migrate individually, and glial cells are not present throughout the chains (Wichterle et al., 1997). Matrigel contains many extracellular matrix molecules, such as collagen, laminin, and heparan sulfate proteoglycans (Kleinman et al., 1982), that may provide sufficient support for SVZ neuronal migration in the absence of glia. Nonetheless, MIA dramatically increases migration from SVZ explants in Matrigel, indicating that SVZ neuronal precursors respond to astrocyte-derived cues even in this extracellular matrix-rich environment.

In vivo studies showed the presence of continuous glial tunnels in the anterior SVZ in adult rodents (Jankovski and Sotelo, 1996; Lois et al., 1996; Peretto et al., 1997), but no GFAP-positive cells associated with the migrating neuronal precursors were observed in early postnatal stages (Law et al., 1999). Although this could be interpreted as evidence that early postnatal chain migration occurs in the absence of astrocytes in vivo, it is likely that immature astrocytes or astrocyte precursors are present in the SVZ but were not detected because of a lack of GFAP expression. GFAP is expressed by mature astrocytes but not in immature ones (Schnitzer and Schachner, 1981; Bovolenta et al., 1984; Pixley and de Vellis, 1984). Moreover, our experiments provide additional support for this. First, SVZ explants in culture express little to no
GFAP at early time points (1-2 DIV), although robust GFAP immunostaining is observed after 3 DIV, suggesting that cells with potential to express GFAP are present in postnatal SVZ explants. Second, analysis of the chains by electron microscopy showed cells with features of astrocytes (type B cells) after 2 DIV, before these cells express GFAP. Together, these findings suggest that immature astrocytes or astrocyte precursors exist in the SVZ of postnatal animals and may promote neuronal chain migration through the secretion of MIA.

Neuronal migration can be viewed as a special set of neuronglia interactions. There is increasing evidence that extracellular signals produced by both cell types are necessary for migration to occur. Migrating neurons, for example, use the growth factor neuregulin to instruct glia to assume a radial morphology and to be supportive of neuronal movement (Anton et al., 1997; Rio et al., 1997). Similarly, this study suggests that glial cells, through the release of MIA, may be instrumental in initiating the movement of neuronal precursors away from the germinal layers, an event for which there is yet no clear explanation. Identification of the protein responsible for this activity, its neuronal receptor, the intracellular signaling mechanisms by which they regulate neuronal movement, and the signals that regulate their expression and function may provide an understanding of basic mechanisms that regulate initial steps in the formation of the vertebrate brain.

\section{REFERENCES}

Aicardi J (1994) The place of neuronal migration abnormalities in child neurology. Can J Neurol Sci 21:185-193.

Alcantara S, Ruiz M, De Castro F, Soriano E, Sotelo C (2000) Netrin 1 acts as an attractive or as a repulsive cue for distinct migrating neurons during the development of the cerebellar system. Development 127:1359-1372.

Anton ES, Marchionni MA, Lee KF, Rakic P (1997) Role of GGF/ neuregulin signaling in interactions between migrating neurons and radial glia in the developing cerebral cortex. Development 124:3501-3510.

Bloch-Gallego E, Ezan F, Tessier-Lavigne M, Sotelo C (1999) Floor plate and netrin-1 are involved in the migration and survival of inferior olivary neurons. J Neurosci 19:4407-4420.

Bovolenta P, Liem RK, Mason CA (1984) Development of cerebellar astroglia: transitions in form and cytoskeletal content. Dev Biol 102:248-259.

Brose K, Bland KS, Wang KH, Arnott D, Henzel W, Goodman CS, Tessier-Lavigne M, Kidd T (1999) Slit proteins bind Robo receptors and have an evolutionarily conserved role in repulsive axon guidance. Neuron 96:795-806.

Brunstrom JE, Gray-Swain MR, Osborne PA, Pearlman AL (1997) Neuronal heterotopias in the developing cerebral cortex produced by neurotrophin-4. Neuron 18:505-517.

Chazal G, Durbec P, Jankovski A, Rougon G, Cremer H (2000) Consequences of neural cell adhesion molecule deficiency on cell migration in the rostral migratory stream of the mouse. J Neurosci 20:1446-1457.

Chen JH, Wen L, Dupuis S, Wu JY, Rao Y (2001) The N-terminal leucine-rich regions in Slit are sufficient to repel olfactory bulb axons and subventricular zone neurons. J Neurosci 21:1548-1556.

Conover JC, Doetsch F, Garcia-Verdugo JM, Gale NW, Yancopoulos GD, Alvarez-Buylla A (2000) Disruption of Eph/ephrin signaling affects migration and proliferation in the adult subventricular zone. Nat Neurosci 3:1091-1097.

Doetsch F, Alvarez-Buylla A (1996) Network of tangential migration in adult mammalian brain. Proc Natl Acad Sci USA 93:14895-14900.

Ebens A, Brose K, Leonardo ED, Hanson Jr MG, Bladt F, Birchmeier C, Barres BA, Tessier-Lavigne M (1996) Hepatocyte growth factor/scatter factor is an axonal chemoattractant and a neurotrophic factor for spinal motor neurons. Neuron 17:1157-1172.

Forsberg-Nilsson K, Behar TN, Afrakhte M, Barker JL, McKay RDG (1998) Platelet-derived growth factor induces chemotaxis of neuroepithelial stem cells. J Neurosci Res 53:521-530.

Garcia-Verdugo JM, Doetsch F, Wichterle H, Lim DA, Alvarez-Buylla A (1998) Architecture and cell types of the adult subventricular zone: in search of the stem cells. J Neurobiol 36:234-248.

Hamanoue M, Takemoto N, Matsumoto K, Nakamura K, Nakajima K, Kohsaka S (1996) Neurotrophic effect of hepatocyte growth factor on central nervous system neurons in vitro. J Neurosci Res 43:554-564. 
$\mathrm{Hu} \mathrm{H}$ (1999) Chemorepulsion of neuronal migration by Slit2 in the developing mammalian forebrain. Neuron 23:703-711.

Hu H, Rutishauser U (1996) A septum-derived chemorepulsive factor for migrating olfactory interneuron precursors. Neuron 16:933-940.

Hu HY, Tomasiewicz H, Magnuson T, Rutishauser U (1996) The role of polysialic acid in migration of olfactory bulb interneuron precursors in the subventricular zone. Neuron 16:735-743.

Jankovski A, Sotelo C (1996) Subventricular zone-olfactory bulb migratory pathway in the adult mouse: cellular composition and specificity as determined by heterochronic and heterotopic transplantation. J Comp Neurol 371:376-396.

Jankovski A, Garcia C, Soriano E, Sotelo C (1998) Proliferation, migration, and differentiation of neuronal progenitor cells in the adult mouse subventricular zone surgically separated from its olfactory bulb. Eur J Neurosci 10:3853-3868.

Jones EG (1995) Cortical development and neuropathology in schizophrenia. Ciba Found Symp 193:277-295.

Kirschenbaum B, Doetsch F, Lois C, Alvarez-Buyalla A (1999) Adult subventricular zone neuronal precursors continue to proliferate and migrate in the absence of the olfactory bulb. J Neurosci 19:2171-2180.

Kleinman HK, McGarvey ML, Liotta LA, Robey PG, Tryggvason K, Martin GR (1982) Isolation and characterization of type IV procollagen, laminin, and heparan sulfate proteoglycan from the EHS sarcoma. Biochemistry 21:6188-6193.

Law AK, Pencea V, Buck CR, Luskin MB (1999) Neurogenesis and neuronal migration in the neonatal rat forebrain anterior subventricular zone do not require GFAP-positive astrocytes. Dev Biol 216:622-634.

Li H, Chen J, Wei W, Fagaly T, Zhou L, Yuan W, Dupuis S, Jiang Z, Nash W, Gick C, Ornitz DM, Wu JY, Rao Y (1999) Vertebrate slit, a secreted ligand for the transmembrane protein roundabout, is a repellent for olfactory bulb axons. Neuron 96:807-818.

Lois C, Alvarez-Buylla A (1994) Long-distance migration in the adult mammalian brain. Science 264:1145-1148.

Lois C, Garcia-Verdugo JM, Alvarez-Buylla A (1996) Chain migration of neuronal precursors. Science 271:978-981.

Luskin MB (1993) Restricted proliferation and migration of postnatally generated neurons derived from the forebrain subventricular zone. Neuron 11:173-189.

O'Connor R, Tessier-Lavigne M (1999) Identification of maxillary factor, a maxillary process-derived chemoattractant for developing trigeminal sensory axons. Neuron 24:165-178.

Ono K, Tomasiewicz H, Magnuson T, Rutishauser U (1994) N-CAM mutation inhibits tangential neuronal migration and is phenocopied by enzymatic removal of polysialic acid. Neuron 13:595-609.

O'Rourke NA (1996) Neuronal chain gangs: homotypic contacts support migration into the olfactory bulb. Neuron 16:1061-1064.

Peretto P, Merighi A, Fasolo A, Bonfanti L (1997) Glial tubes in the rostral migratory stream of the adult rat. Brain Res Bull 42:9-21.

Pixley SK, de Vellis J (1984) Transition between immature radial glia and mature astrocytes studied with a monoclonal antibody to vimentin. Brain Res 317:201-209.

Powell EM, Mars WM, Levitt P (2001) Hepatocyte growth factor/scatter factor is a motogen for interneurons migrating from the ventral to dorsal telencephalon. Neuron 30:79-89.

Rando TA, Blau HM (1994) Primary mouse myoblast purification, characterization, and transplantation for cell-mediated gene therapy. J Cell Biol 125:1275-1287.

Raymond AA, Fish DR, Stevens JM, Sisodiya SM, Alsanjari N, Shorvon SD (1994) Subependymal heterotopia: a distinct neuronal migration disorder associated with epilepsy. J Neurol Neurosurg Psychiatry 57:1195-1202.

Rio C, Rieff HI, Qi P, Khurana TS, Corfas G (1997) Neuregulin and erbB receptors play a critical role in neuronal migration. Neuron 19:39-50.

Rousselot P, Lois C, Alvarez-Buylla A (1995) Embryonic (PSA) N-CAM reveals chains of migrating neuroblasts between the latera ventricle and the olfactory bulb of adult mice. J Comp Neurol 351:51-61.

Schnitzer J, Schachner M (1981) Developmental expression of cell typespecific markers in mouse cerebellar cells in vitro. J Neuroimmunol 1:471-487.

Song H, Ming G, He Z, Lehmann M, McKerracher L, Tessier-Lavigne M, Poo M (1998) Conversion of neuronal growth cone responses from repulsion to attraction by cyclic nucleotides. Science 281:1515-1518

Stein E, Tessier-Lavigne M (2001) Hierarchical organization of guidance receptors: silencing of netrin attraction by slit through a Robo/ DCC receptor complex. Science 291:1928-1938.

Tessier-Lavigne M, Placzek M, Lumsden AGS, Dodd J, Jessell TM (1988) Chemotropic guidance of developing axons in the mammalian central nervous system. Nature 336:775-778.

Thewke DP, Seeds NW (1996) Expression of hepatocyte growth factor/ scatter factor, its receptor, c-met, and tissue-type plasminogen activator during development of the murine olfactory system. J Neurosci 16:6933-6944.

Tomasiewicz H, Ono K, Yee D, Thompson C, Goridis C, Rutishauser U, Magnuson T (1993) Genetic deletion of a neural cell adhesion molecule variant (N-Cam-180) produces distinct defects in the central nervous system. Neuron 11:1163-1174.

Uher BF, Golden JA (2000) Neuronal migration defects of the cerebral cortex: a destination debacle. Clin Genet 58:16-24.

Wichterle H, Garcia-Verdugo JM, Alvarez-Buylla A (1997) Direct evidence for homotypic, glia-independent neuronal migration. Neuron 18:779-791.

Wu W, Wong K, Chen JH, Jiang ZH, Dupuis S, Wu J, Rao Y (1999) Directional guidance of neuronal migration in the olfactory system by the protein Slit. Nature 400:331-336.

Yee KT, Simon HH, Tessier-Lavigne M, O'Leary DM (1999) Extension of long leading processes and neuronal migration in the mammalian brain directed by the chemoattractant netrin-1. Neuron 24:607-622. 